\title{
Stimulation of 1-aminocyclopropane-1-carboxylic acid-dependent ethylene production in detached rice leaves by methyl jasmonate
}

\author{
Chang Mei Chou and Ching Huei Kao
}

\author{
Department of Agronomy, National Taiwan University, Taipei, Taiwan (Republic of China)
}

(Received December 2nd, 1991; revision received January 30th, 1992; accepted January 31st, 1992)

\begin{abstract}
Effects of methyl jasmonate (JA-Me) on ethylene production in detached rice leaves were investigated. Without 1-aminocyclopropane-1-carboxylic acid (ACC) pretreatment, JA-Me had no effect on ethylene production. However, JA-Me markedly stimulated ethylene production in detached rice leaves pretreated with a saturating concentration of ACC. JA-Me stimulated ACCdependent ethylene production within $2 \mathrm{~h}$ of its application, a result suggesting that $\mathrm{JA}$-Me enhances ACC-dependent ethylene production directly. $\mathrm{CoCl}_{2}$ at $1 \mathrm{mM}$ inhibited ACC-dependent ethylene production stimulated by JA-Me, suggesting that ethylene production from ACC is mediated by the ethylene-forming enzyme. Cycloheximide also inhibited ACC-dependent ethylene production induced by JA-Me, indicating that sustained synthesis of the ethylene-forming enzyme is required. JA-Me stimulated ACC-dependent ethylene production in detached leaves that had been aged for 1, 2 and 3 days. However, aged leaves were less responsive to JA-Me in terms of ACC-dependent ethylene production. Abscisic acid was found to inhibit ACC-dependent ethylene production stimulated by JA-Me.
\end{abstract}

Key words: abscisic acid; 1-aminocyclopropane-1-carboxylic acid; ethylene; methyl jasmonate; Oryza sativa

\section{Introduction}

Methyl jasmonate (JA-Me) and jasmonic acid are endogenous substances which have been identified in many plants [1-3]. JA-Me and jasmonic acid were found to be powerful promoters of leaf senescence [2,4-6]. Ethylene has been shown to be involved in the regulation of leaf senescence [7-9]. Recently, Saniewski et al. [10-11] reported that JA-Me stimulated ethylene biosynthesis in tomato fruits. However, this is the only plant system so far that has been used to examine the effect of JA-Me on ethylene biosynthesis. It is not known whether other plant or tissue systems also show a similar effect of JA-Me on ethylene production. For this

Correspondence to: Ching Huei Kao, Department of Agronomy, National Taiwan University, Taipei, Taiwan, Republic of China.

Abbreviations: ABA, abscisic acid; ACC, 1-aminocyclopropane-1-carboxylic acid; JA-Me, methyl jasmonate. reason we investigated the effect of JA-Me on ethylene production in detached rice leaves.

\section{Materials and Methods}

Seedlings of rice (Oryza sativa L. cv. Taichung Native 1) seedlings were grown in hydroponic culture as described previously [12]. Unless otherwise indicated, ACC-dependent ethylene production was determined. For the determination of ACC-dependent ethylene production, 10 leaf segments, weighing $45 \mathrm{mg}$, were pretreated with a saturating concentration of ACC $(10 \mathrm{mM})$ in water and then treated with various compounds in water for various periods of time as indicated in the figure and table legends. Pretreatment and treatment were carried out at $27^{\circ} \mathrm{C}$ in darkness. At the time indicated, leaf segments were placed vertically in test tubes which were closed with rubber stoppers and incubated in darkness at $27^{\circ} \mathrm{C}$ for 
$1 \mathrm{~h}$. The ethylene in the gas phase of the closed tubes was determined by analysis of $1 \mathrm{ml}$ of sample withdrawn with a hypodermic syringe as described elsewhere [9]. Absolute levels of ethylene varied among experiments because of seasonal effects. However, the patterns of responses to JA-Me were reproducible. Each treatment had 4 replicates. All experiments described here were repeated at least twice. Similar results and identical trends were obtained each time. The data reported here were from a single experiment.

\section{Results and Discussion}

The effects of the concentration of JA-Me on ACC-dependent ethylene production in detached rice leaves in darkness are presented in Fig. 1. JAMe markedly promoted ACC-dependent ethylene production in detached rice leaves. Increasing JAMe concentration from 0.45 to $45 \mu \mathrm{M}$ progressively enhanced ACC-dependent ethylene production by detached rice leaves. However, ACC-dependent ethylene production declined at a JA-Me concen-

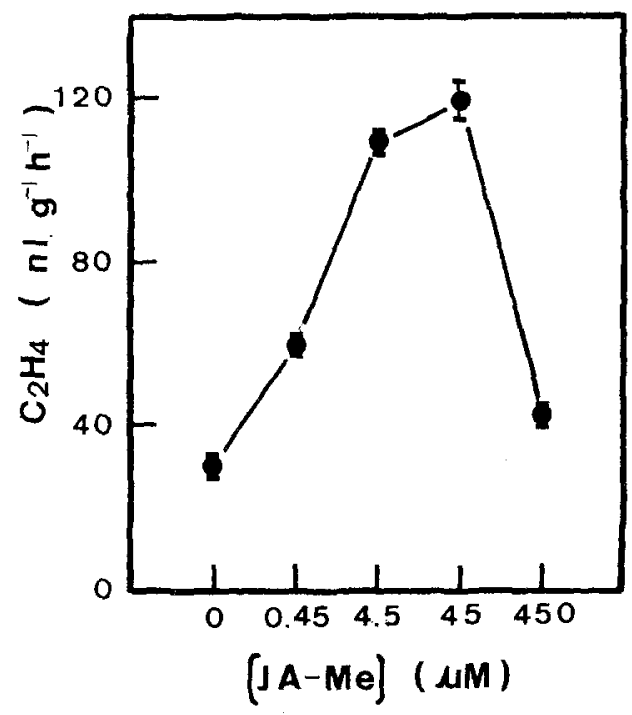

Fig. 1. Effect of JA-Me concentration on ACC-dependent ethylene production in detached rice leaves. Detached rice leaves were pretreated with $10 \mathrm{mM} \mathrm{ACC}$ in water for $2 \mathrm{~h}$ and then treated with various concentrations of aqueous JA-Me in darkness. Ethylene production was assayed after $6 \mathrm{~h}$ of treatment. Bars indicate standard errors.

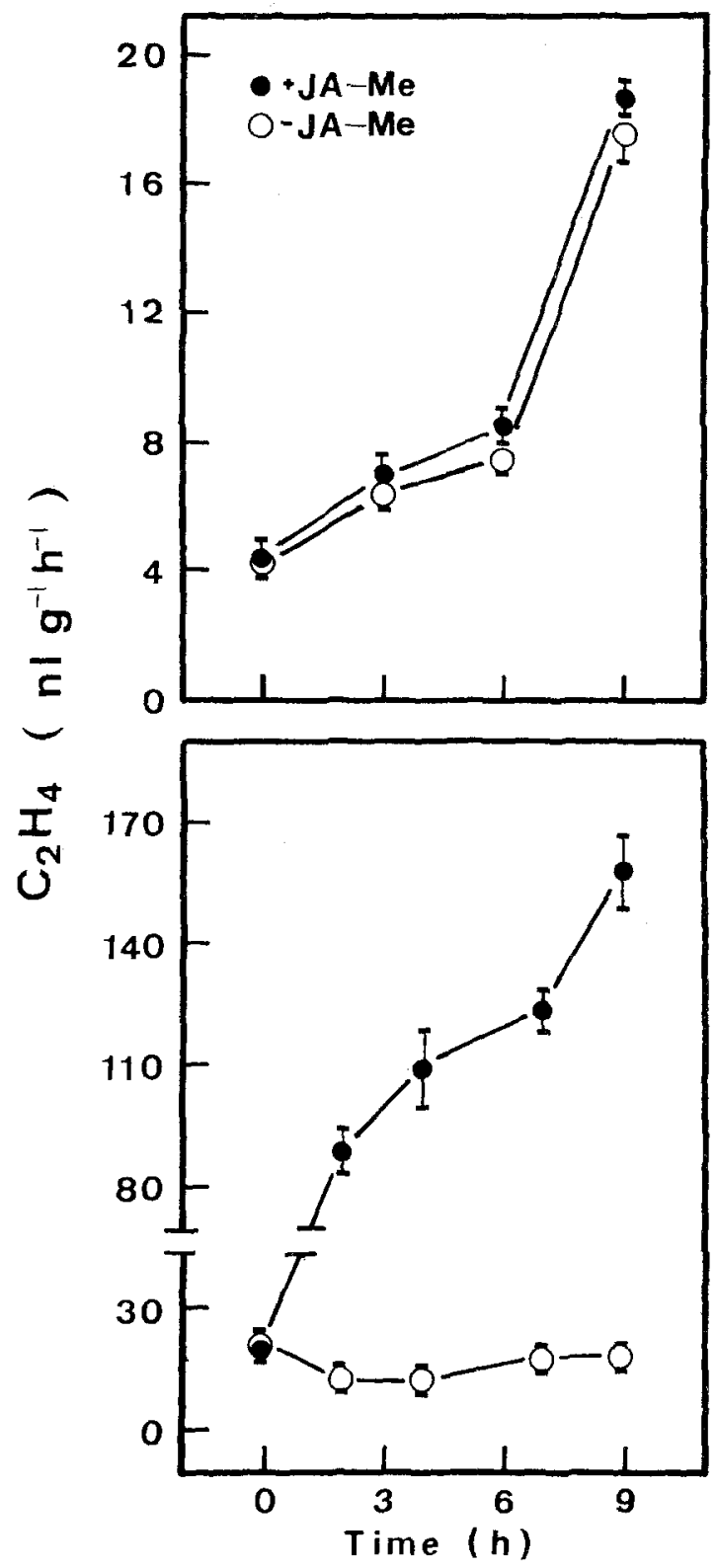

Fig. 2. Changes with time in rates of basal (upper) and and ACC-dependent (lower) ethylene production in detached rice leaves treated with JA-Me. For basal ethylene production, detached leaves were treated with either water or $45 \mu \mathrm{M} \mathrm{JA}-\mathrm{Me}$ in water in darkness. To determine ACC-dependent ethylene production, detached rice leaves were pretreated with a saturating concentration of ACC (10 mM) in water for $2 \mathrm{~h}$ and then treated with either water or $45 \mu \mathrm{M} \mathrm{JA}-\mathrm{Me}$ in water in darkness. Ethylene production was assayed at the times indicated. Bars indicate standard errors. 
tration of $450 \mu \mathrm{M}$. The ability of JA-Me to stimulate ACC-dependent ethylene production was also observed when illumination (16.7 $\mu \mathrm{mol} / \mathrm{m}^{2}$ per $\mathrm{s}$, photosynthetic photon flux, provided by Gro-lux fluorescent lamp) was used (data not shown).

Ethylene production was stimulated in tomatoes by JA-Me in the absence of exogenous ACC [10-11]. However, we could not detect any effect of JA-Me on ethylene production without ACC pretreatment (Fig. 2, upper).

JA-Me stimulated ACC-dependent ethylene production was detected within $2 \mathrm{~h}$ of its application (Fig. 2, lower). Increasing duration of treatment with JA-Me from 2 to $9 \mathrm{~h}$ progressively enhanced ACC-dependent ethylene production by detached rice leaves. The observation that the stimulation of ACC-dependent ethylene production could be detected immediately after application of JA-Me suggests that JA-Me directly enhances ACC-dependent ethylene production.

In order to determine if ethylene production from ACC is mediated by ethylene-forming enzyme in detached rice leaves treated with JA-Me, the effect of $1 \mathrm{mM} \mathrm{CoCl}$, which inhibits the conversion of ACC to ethylene [12], was studied (Table I). Ethylene formation was inhibited by $\mathrm{CoCl}_{2}$ in detached rice leaves treated with JA-Me, indicating that detached rice leaves treated with JA-Me formed ethylene from ACC through ethylene-forming enzyme. Cycloheximide also inhibited ACC-dependent ethylene production by JA-Me (Table I), suggesting that sustained synthesis of the ethylene-forming enzyme is required

Table I. Effects of $\mathrm{CoCl}_{2}$ and cycloheximide (CHI) on ACCdependent ethylene production induced by methyl jasmonate (JA-Me) in detached rice leaves. Detached rice leaves were pretreated with ACC (10 mM) in water for $2 \mathrm{~h}$ and then treated with aqueous JA-Me $(45 \mu \mathrm{M})$ in the absence or presence of $\mathrm{CoCl}_{2}(1 \mathrm{mM})$ or $\mathrm{CHI}(50 \mu \mathrm{M})$. Ethylene was assayed after 3 $h$ of treatment in darkness. Means \pm S.E. are shown.

\begin{tabular}{ll}
\hline Treatment & Ethylene $(\mathrm{nl} / \mathrm{g}$ per h) \\
\hline No Additions & $23.0 \pm 1.4$ \\
$+\mathrm{JA}-\mathrm{Me}$ & $93.2 \pm 2.3$ \\
$+\mathrm{JA}-\mathrm{Me}+\mathrm{CoCl}_{2}$ & $29.1 \pm 2.4$ \\
$+\mathrm{JA}-\mathrm{Me}+\mathrm{CHI}$ & $35.7 \pm 0.7$ \\
\hline
\end{tabular}

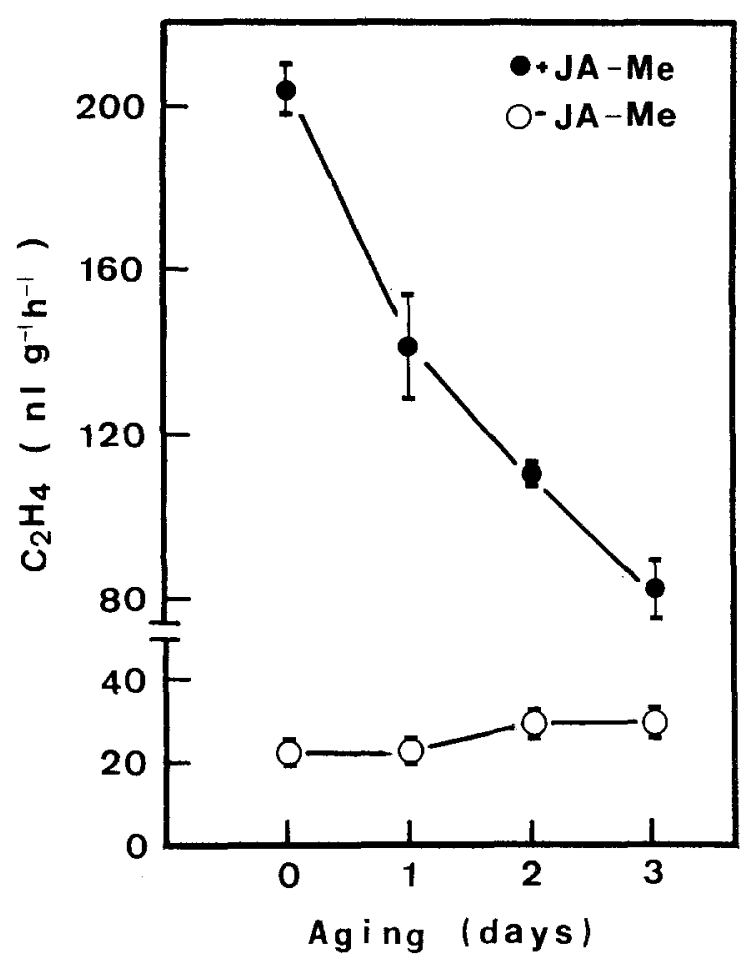

Fig. 3. Effect of aging on methyl jasmonate (JA-Me)stimulated ACC-dependent ethylene production in detached rice leaves. Detached rice leaves, aged for 1,2 , or 3 days in water in darkness, were pretreated with $10 \mathrm{mM} \mathrm{ACC}$ in water and then treated with either water or $45 \mu \mathrm{M} \mathrm{JA}-\mathrm{Me}$ in water in darkness. Ethylene production was assayed after $6 \mathrm{~h}$ of treatment. Bars indicate standard errors.

for JA-Me-stimulated ACC-dependent ethylene production.

To study the effects of aging, segments of rice leaves were incubated in water in darkness for 1 , 2 , or 3 days before the start of the experiment. Segments of rice leaves aged for 1, 2 or 3 days all exhibited JA-Me-stimulated ACC-dependent ethylene production (Fig. 3). However, aged leaf segments were less responsive to JA-Me in terms of ACC-dependent ethylene production. Increasing the duration of aging resulted in a decrease of ACC-dependent ethylene production induced by JA-Me. Untreated segments of leaves produced low levels of ACC-dependent ethylene regardless of the duration of aging (Fig. 3).

Jasmonic acid is similar to ABA both chemically 


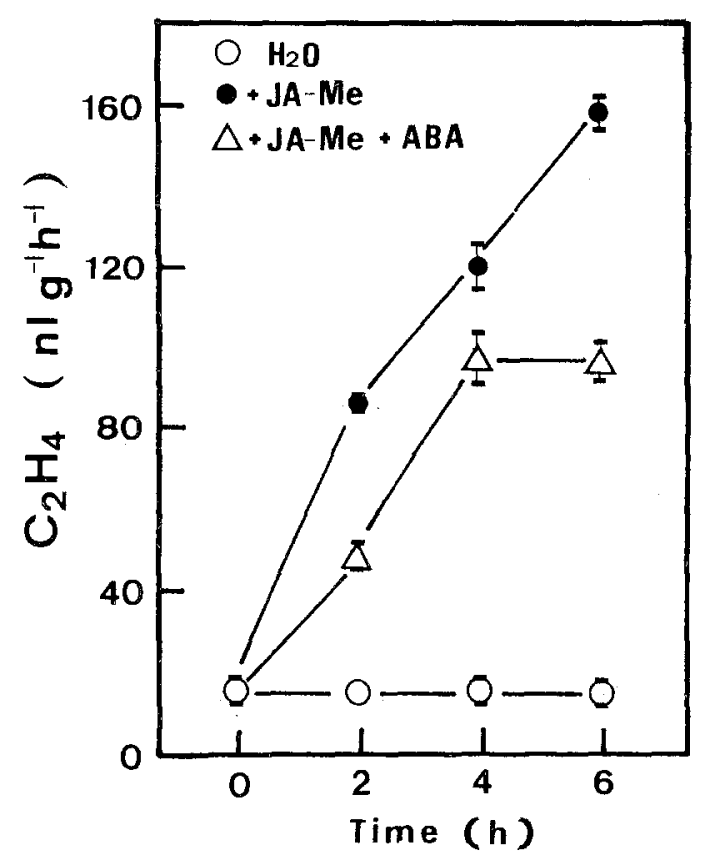

Fig. 4. Effect of methyl jasmonate (JA-Me) in the absence or presence of $\mathrm{ABA}$ on ACC-dependent ethylene production in detached rice leaves. Detached rice leaves were pretreated with $10 \mathrm{mM} \mathrm{ACC}$ in water and then treated with either water, JA$\mathrm{Me}(45 \mu \mathrm{M})$ in water or JA-Me $(45 \mu \mathrm{M})+\mathrm{ABA}(45 \mu \mathrm{M})$ in water in darkness. Ethylene production was assayed at the times indicated. Bars indicate standard errors.

and biologically [13]. Both compounds are ketoacids with similar molecular weights, solubility properties and $\mathrm{pKs}$ and in some tissues, jasmonic acid causes an inhibition of growth that often mimics the effect of ABA [14]. To determine whether JA-Me interacts with ABA in regulating ACC-dependent ethylene production, the effect of $\mathrm{JA}-\mathrm{Me}$ in the absence or presence of $\mathrm{ABA}$ on ACC-dependent ethylene production was examined. ACC-dependent ethylene production was found to be significantly inhibited by ABA (Fig. 4). This inhibition is probably not due to the inhibition of JA-Me uptake by ABA, because detached rice leaves pretreated with $A B A$ also resulted in an inhibition of ACC-dependent ethylene production stimulated by JA-Me (data not shown). ABA has also been reported to inhibit stress-induced ethylene production [15-17].

It has been shown that ABA levels increase dur- ing aging of segments of oat leaves [18]. If ABA levels increase similarly in aged rice leaves, then the decrease in ACC-dependent ethylene production stimulated by JA-Me in aged rice leaves could result from $\mathrm{ABA}$-mediated inhibition.

\section{Acknowledgement}

This research was supported financially by the National Science Council, Republic of China under Grant NSC 81-0115-C002-01-162B. The assistance of $\mathrm{Mr}$. Chien Teh Chen is gratefully acknowledged.

\section{References}

1 L. Crabalona, Presence of levorotatory methyl jasmonate, methyl cis-2(2-penten-1-yl)-3-oxocyclopentenyl acetate, in the essential oil of Tunesian rosemary. CR Acad. Sci. (Paris) Ser. C, 264 (1967) 2074-2076.

2 J. Ueda and J. Kato, Isolation and identification of a senescence-promoting substance from wormwood (Artemisia absinthum L.). Plant Physiol., 66 (1980) 246-249.

3 A. Meyer, O. Miersch, C. Buttner, W. Dathe and G. Sembdner, Occurrence of the plant growth regulator jasmonic acid in plants. J. Plant Growth Regul., 3 (1984) $1-8$.

4 J. Ueda and J. Kato, Promotive effect of methyl jasmonate on oat leaf senescence in the light. Z. Pflanzenphysiol., 103 (1981) 357-359.

5 J. Ueda and J. Kato, Inhibition of cytokinin-induced plant growth by jasmonic acid and its methyl ester. Physiol. Plant., 54 (1982) 249-252.

6 J. Ueda, J. Kato, H. Yamane and N. Takahashi, Inhibitory effect of methyl jasmonate and its related compounds on kinetin-induced retardation of oat leaf senescence. Physiol. Plant., 52 (1981) 305-309.

7 N. Aharoni and M. Lieberman, Ethylene as a regulator of senescence in tobacco leaf discs. Plant Physiol., 64 (1979) $801-804$.

8 S. Gepstein and K.V. Thimann, The role of ethylene in the senescence of oat leaves. Plant Physiol., 68 (1981) $349-354$.

9 C.H. Kao and S.F. Yang, Role of ethylene in the senescence of detached rice leaves. Plant Physiol, 73 (1983) 881-885.

10 M. Saniewski, J. Nowacki and J. Czapski, The effect of methyl jasmonate on ethylene production and ethyleneforming enzyme activity in tomatoes. J. Plant Physiol., 129 (1987) $175-180$.

11 M. Saniewski, H. Urbanek and J. Czapski, Effects of methyl jasmonate on ethylene production, chlorophyll degradation, and polygalacturonase activity in tomatoes. J. Plant Physiol., 127 (1987) 177-181. 
12 Y.B. Yu and S.F. Yang, Auxin induced ethylene production and its inhibition by aminoethoxyvinylglycine and cobalt ion. Plant Physiol., 64 (1979) 1074-1077.

13 R.A. Weidhase, H.M. Kramell, J. Lehmann, H.W. Liebisch, W. Lerbs and B. Parthier, Methyl jasmonate induced changes in the polypeptide pattern of senescing barley leaf segments. Plant Sci., 51 (1987) 177-186.

14 J.M. Anderson, Membrane-derived fatty acids as precursors to second messengers, in: W.F. Boss and D.J. Morre (Eds.), Second Messengers in Plant Growth and Development, A.R. Liss, New York, 1989, pp. 181-212.

15 Y.H. Jeong, H. Nakamura and Y. Ota, Physiological studies on phytochemical oxidant injury to rice plants II. Effect of abscisic acid on ozone injury and ethylene pro- duction in rice plants. Nippon Sakumotsu Gakkai Kiji, 50 (1981) 560-565.

16 T.A. McKeon, N.E. Hoffman and S.F. Yang, The effect of plant hormone pretreatments on ethylene production and synthesis of 1-aminocyclopropane-1-carboxylic acid in water-stressed wheat leaves. Planta, 63 (1979) 142-145.

17 S.T.C. Wright, The effect of plant growth regulator treatments on the levels of ethylene emanating from excised turgid and wilted wheat leaves. Planta, 148 (1980) $381-388$.

18 S. Gepstein and K.V. Thimann, Changes in the abscisic acid content of oat leaves during senescence. Proc. Natl. Acad. Sci. U.S.A., 77 (1980) 2050-2053. 